\title{
DEVELOPING NEW CHARGED SYSTEM SEARCH-BASED ALGORITHM: APPLICATION IN THE TIME-COST TRADE-OFF PROBLEMS
}

\author{
M.K. Sharbatdar \\ Faculty of Civil Engineering, Semnan University, Semnan, Iran \\ msharbatdar@semnan.ac.ir \\ S. Talatahari \\ Engineering, University of Tabriz, Tabriz, Iran Department of Civil \\ talatahari@tabrizu.ac.ir \\ M.R. Mousavi \\ Faculty of Civil Engineering, Semnan University, Semnan, Iran \\ rm_Mousavi@semnan.ac.ir
}

\begin{abstract}
The trade-off between the total cost and project duration is one of the most important parameters of construction project planning. There are various methods to optimize time-cost trade-off problems. Mathematical programming models as one type of them cannot solve large and complex networks effectively. On the other hand, although the meta-heuristics algorithms in many cases can find a complete set of solutions but to optimize the time-cost trade-off problems in very massive construction projects they need to spend a lot of time, so existence a powerful algorithm with higher convergence rate is necessary. In this paper new procedures MAWA-CSS and SMOCSS are introduced to generalize the well-known CSS algorithm for solving TCTP optimization problem and all multi-objective optimization problems in discrete and continuous search space. The overall structure of SMOCSS algorithm is similar to the MOPSO and to determine the Charge magnitude of particles a new simple method is introduced. The proposed method is examined for different test functions and the results are compared to the results of two well-known multiobjective algorithms (NSGA-II and MOPSO). In addition, two example of time-cost optimization problem (Feng and Zheng network with 18 and 7 activities respectively) are used to evaluate the performance of the proposed algorithms. The results indicate that the SMOCSS algorithm has the ability to find out the optimal solution and define the Pareto front as well in reasonable time. Hence the proposed approach in this paper is much adaptive and suitable for tackling TCTP, which is useful and beneficial for decision-making on the trade-off between project duration and total cost.
\end{abstract}

Keywords: Time-Cost Trade-off Problems (TCTP), multi-objective optimization, meta-heuristics algorithms, Charged System Search (CSS)

\section{INTRODUCTION}

The complexities and difficulties of a construction project causes changes in costs and timing of the project in the implementation phase. With the rapid expansion of the use of various systems project delivery, time is a determining factor in the evaluation of tenders and the manufacturing process. Therefore, construction managers not focus just on reducing costs but the project execution time is also so important. Forasmuch the compression of time will inevitably lead to a gradual increase in direct costs of the project, in project management, achieving optimal time-cost of the project becomes very important [1-3].

Several mathematical models such as linear programming [4, 5], integer programming, or dynamic programming $[2,6,7]$ and LP/IP hybrid models are used to solve TCTPs [8, 9]. These methods are not suitable for large scale projects and cannot find all possible solutions [10]. Because of the population-based 
nature of multi-objective evolutionary algorithms and their ability to find multiple optima simultaneously $[11,12]$, several different algorithms have been suggested in recent years to solve TCTP which can include Non-Dominated Sorting Genetic Algorithm (NSGA_II) [3, 10] Particle Swarm Optimization (PSO) [13], Adaptive Weighted Approach (AWA) and etc. [14-16]. In this study, the main contribution is on introducing different multi-objective CSS algorithms that, in addition to simplicity, they are capable on finding global optimum solutions and have high convergence rate.

The charged System Search (CSS) is one of the recently introduced single-objective optimization algorithm which have been used in science and engineering optimization problems successfully. This method mixes the governing motion from Newtonian mechanics and the governing Coulomb law from physics. In the CSS algorithm, each possible solution corresponds to a charged particle (CP) which can impose an attractive electric force on other CPs according to Coulomb's law. The next position of each CP is determined by Calculating the resultant forces acting on the CP and applying the kinematic equations [17-19]. The results of this algorithm compared with each other and with some other well-known multi-objective optimization methods. Also we will use the well-known these approaches to solve time-cost problem.

\section{PRELIMINARIES}

\section{BASIC CONCEPTS IN MULTI-OBJECTIVE OPTIMIZATION MOO}

For better understanding of the Multi-Objective Problems (MOPs), acquaintance with the following concepts are necessary [20]:

General Multi-objective Optimization Problem: The aim in a multi-objective optimization for minimization problem is finding a vector $x=\left(x_{1}, x_{2}, \ldots, x_{n}\right)$ which satisfies $k$ inequality constraints as:

$$
q_{i}(x) \leq 0 \quad(i=1,2, \ldots, k)
$$

and $l$ equality constraints:

$$
h_{i}(x)=0 \quad(i=1,2, \ldots, l)
$$

and minimizes the vector function

$$
\operatorname{Min}_{x \in \Omega} F(x)=\left\{f_{1}(x), f_{2}(x), \ldots, f_{m}(x)\right\}
$$

Where $m$ is the number of objectives and $\Omega$ is a set of the decision vector.

Pareto Dominance: A vector $\boldsymbol{u}=\left(u_{1}, u_{2}, \ldots, u_{n}\right)$ dominates vector $\boldsymbol{v}=\left(v_{1}, v_{2}, \ldots, v_{n}\right)($ denoted by $\boldsymbol{u}<\boldsymbol{v})$ if and only if $\boldsymbol{u}$ is partially less than $\boldsymbol{v}$, i.e.,

$\forall i \in\{1,2, \ldots, n\}, u_{i} \leq v_{i} \wedge \exists i \in\{1,2, \ldots, n\}: u_{i}<v_{i}$.

Pareto Optimal: A solution $x \in \Omega$ is Pareto Optimal with respect to $\Omega$ if and only if there is no $x^{\prime} \in \Omega$ for which $\boldsymbol{v}=\left(f_{1}\left(x^{\prime}\right), f_{2}\left(x^{\prime}\right), \ldots, f_{n}\left(x^{\prime}\right)\right)$ dominates $\boldsymbol{u}=\left(f_{1}(x), f_{2}(x), \ldots, f_{n}(x)\right)$.

Pareto Optimal Set: For a given MOP, $F(x)$, the Pareto Optimal Set, $P$, is defined as $P=\left\{x \in \Omega \mid \neg \exists x^{\prime} \in \Omega F\left(x^{\prime}\right)<F(x)\right\}$.

Pareto Optimal Front: For a given MOP, $F(x)$, and Pareto Optimal Set, $P$, the Pareto Front $P F$ is defined as $P F=\{\boldsymbol{u}=F(x) \mid x \in P\}$. 
A solution is said to be Pareto Optimal if it is not dominated by any other solutions in the search space, also termed as non-dominated solution. In this paper, we distinguish the real Pareto Optimal front, termed $P F_{\text {real }}$, and the final set of non-dominated solutions obtained by a multi-objective optimization algorithm, termed $P F_{\text {algorithm }}$ as defined by the aim of the multi-objective optimization algorithms is to find a well uniformly distributed $P F_{\text {algorithm }}$ that approximates $P F_{\text {algorithm }}$ as close as possible.

\section{CHARGED SYSTEM SEARCH}

Charged System Search (CSS) algorithm, introduced by Kaveh and Talatahari [17], is based on electrostatic and Newtonian mechanics laws. In the CSS algorithm, charged particles (CPs) are assumed to be the candidate solutions. Charged particle are affected by the electric field that created by other particles. The amount of force exerted on each CP, are obtained using the electrostatic rules. Also, the motion of each $\mathrm{CP}$ is determined by the rules of Newtonian mechanics and the charge magnitude of each particle will be determined according to the value of the objective function [17-19]. Fig. 1 shows the pseudo-code of the CSS algorithm.

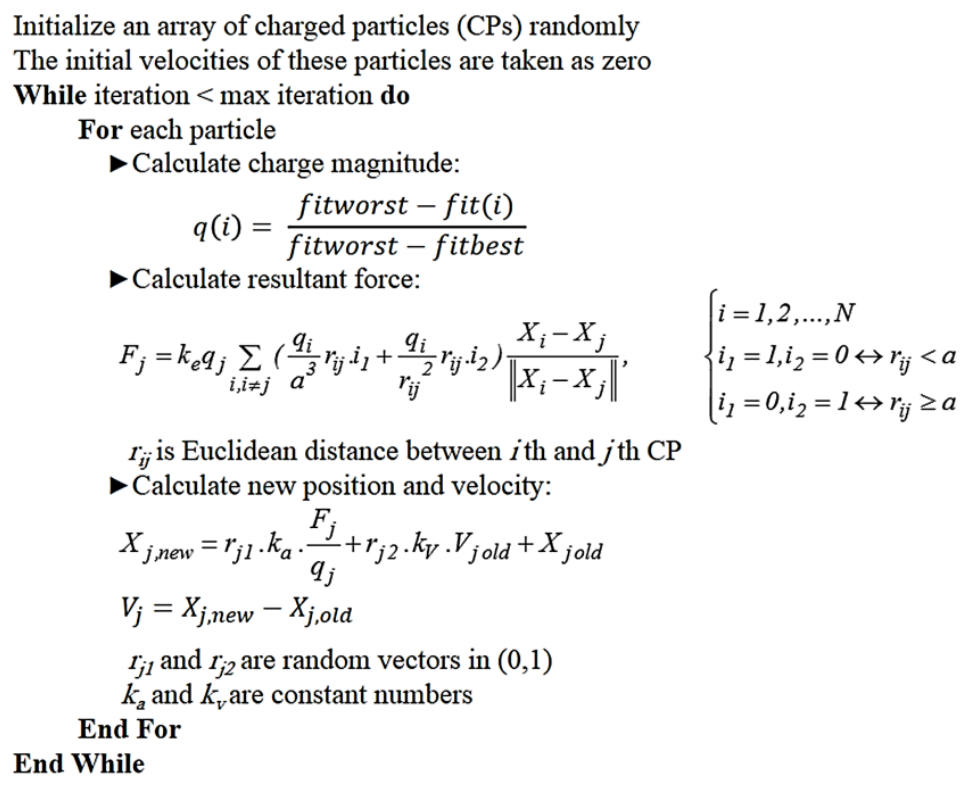

Fig. 1: pseudo-code of the CSS algorithm.

\section{TCTP PROBLEM FORMULATION}

In the trade-off between the total cost and project duration problems (TCTP), there are twin objectives to be minimized: the project time and its cost. In a project, a two-objective optimization problem should be solved as follows:

$\left\{\begin{array}{l}\min \quad T \\ \min \quad C\end{array}\right.$

Where $T$ is the total project time and $C$ is the total project cost that they are defined as:

$$
\left\{\begin{array}{l}
T=\max _{L_{k} \in L}\left[\sum_{i \in L_{k}} t_{i}^{(k)} x_{i}^{(k)}\right] \\
C=\sum_{i \in A} d c_{i}^{(k)} x_{i}^{(k)}+T \times i c_{i}^{(k)}
\end{array}\right.
$$


Where $A$ is the number of activities, $t_{i}^{(k)}$ represents the duration of activity $i$ when performing the $k$ th option, $x_{i}^{(k)}$ stands for the index variable of activity $i$ when performing the $k$ th option. If $x_{i}^{(k)}=1$ then the activity $i$ perform the $k$ th option. The sum of index variables of all options should be equal to 1 . $L_{k}$ means the activity sequence on the $k$ th path, and $L_{k}=\left\{i_{1 k}, i_{2 k}, \ldots, i_{n k}\right\}$ where $i_{j k}$ represents the sequence number of activity $j$ on the $k$ th path. $L$ stands for the set of all paths of a network, and $L=\left\{L_{k} k=1,2, \ldots, m\right\}$, where $m$ symbolizes the number of all paths of a network. $d c_{i}^{(k)}$ and $i c_{i}^{(k)}$ represent the direct and indirect cost of activity $i$ when performing the $k$ th option, respectively $[3,13,16]$.

\section{NEW SIMPLE MULTI-OBJECTIVE CHARGED SYSTEM SEARCH (SMOCSS)}

Due to the high convergence and large capability of CSS algorithm [17] we produced a new simple multiobjective optimization algorithm based on CSS algorithm (SMOCSS) that, in addition to simplicity, is capable of finding global optimum solutions and have high convergence rate [21-23]. The flowchart of this algorithm is shown schematically in Fig.2.

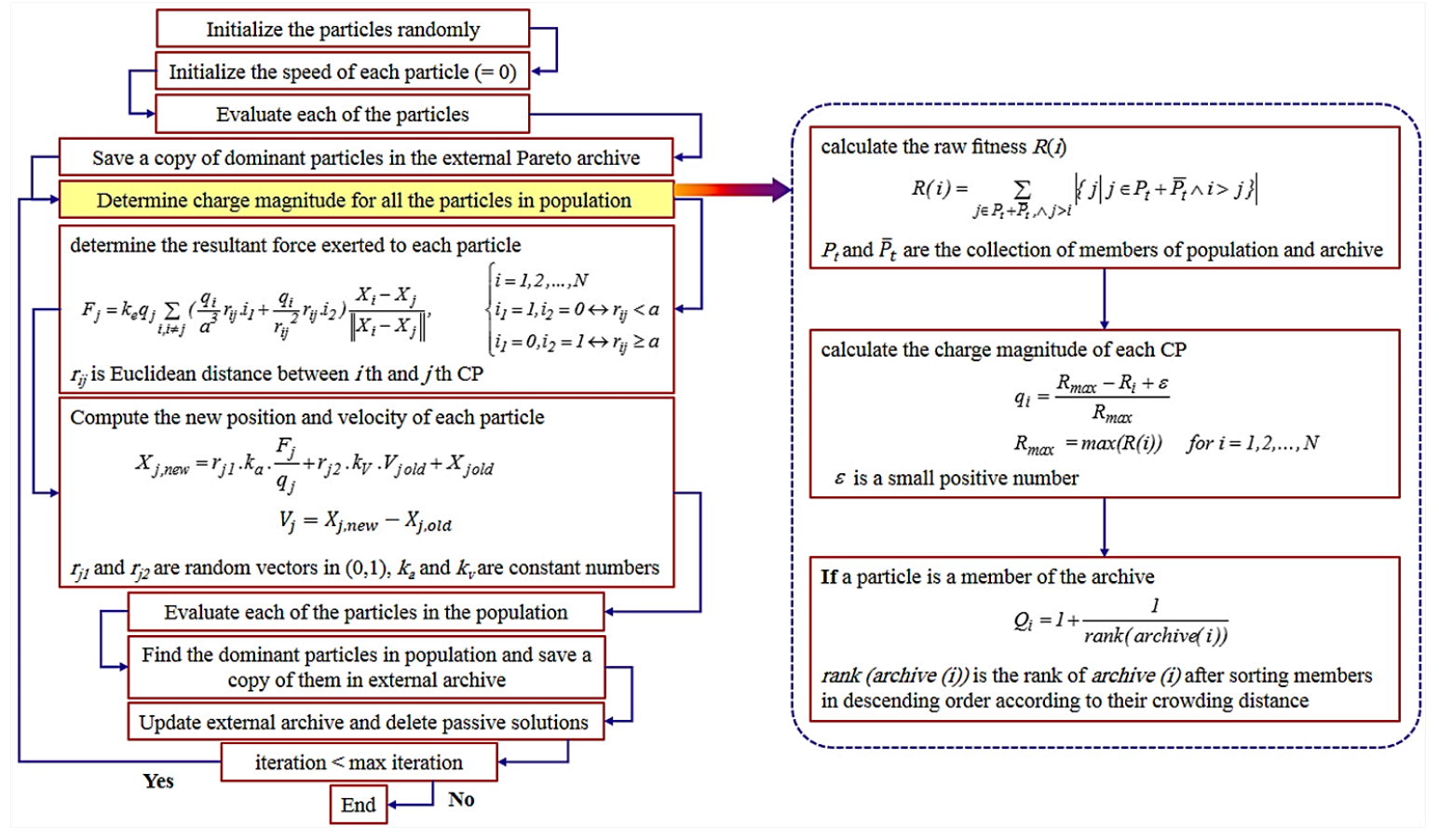

Fig.2: flowchart of SMOCSS algorithm

\section{CHARGED SYSTEM SEARCH FOR MODIFIED ADAPTIVE WEIGHT APPROACH (MAWA- CSS-TCTP)}

TCTP problem is a multi-objective optimization problem. One positive trend to solve this problem is Modified Adaptive Weight Approach [16]. In this paper based on Charged System Search algorithm and modified adaptive weighting method a model is developed and pareto front is achieved. The main steps of MAWA-CSS algorithm for TCTP are described as follows:

1) Randomly generation of initial solutions.

2) Computing the project duration and total cost from Eq.2. 
3) Choosing the dominant answers and adding to the Pareto solution set

4) Calculating the alternative fitness value as:

$$
f(X)=w_{t} \frac{Z_{t}-Z_{t}^{\min }+\gamma}{Z_{t}^{\max }-Z_{t}^{\min }+\gamma}+w_{c} \frac{Z_{c}-Z_{c}^{\min }+\gamma}{Z_{c}^{\max }-Z_{c}^{\min }+\gamma}
$$

Which $Z_{t}^{\text {min }}$ and $Z_{t}^{\max }$ as the minimal value and maximal value for the objective of duration respectively, $Z_{c}^{\text {min }}$ and $Z_{c}{ }^{m a x}$ as the maximal value and minimal value for the objective of cost respectively, $w_{t}$ and $w_{c}$ are adaptive weight for the criterion of time and cost respectively that can be calculate by considering this conditions as:

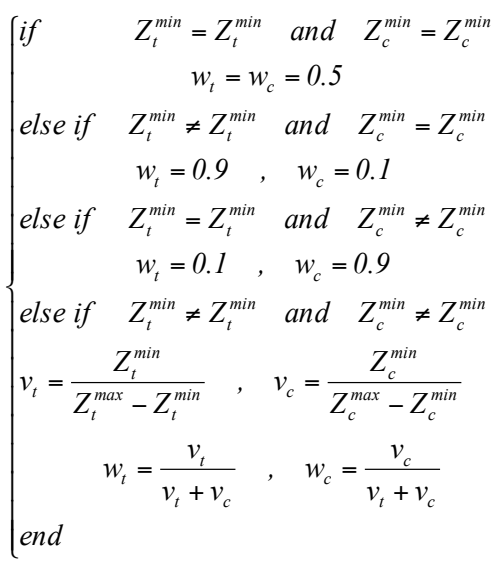

5) Determine charge magnitude for all the particles in population as:

$q_{i}=\frac{\text { fit }(i)-\text { fitworst }}{\text { fitbest }- \text { fitworst }}, \quad i=1,2, \ldots, N$

Where fitbest and fitworst are the so far best and the worst fitness of all particles; fit(i) represents the objective function value or the fitness of the agent $i$, and $N$ is the total number of particles.

6) Initialize the $\mathrm{F}$ vector (resultant force vector acted on each particle) and determine the resultant force exerted to each particle.

7) Compute the new position and velocity of each particle.

8) Maintain the particles within the search space [22].

9) Repeating step 2 to step 8 until the generated Pareto solutions are repeated and there is no new solution in the generated set.

\section{NUMERICAL EXAMPLES}

\section{PERFORMANCE METRICS}

In order to provide a quantitative assessment for the performance of an MO optimizer, three issues are often taken into consideration [24]:

a) The distance of the resulting non-dominated set to the Pareto-optimal front should be minimized. 
b) A good (in most cases uniform) distribution of the solutions found is desirable. The assessment of this criterion might be based on a certain distance metric.

c) The extent of the obtained non-dominated front should be maximized, i.e., for each objective, a wide range of values should be covered by the non-dominated solutions.

Comparative studies performed by researchers such as [23-26] made use of a suite of unary performance metrics pertinent to the optimization goals of proximity, distribution, and diversity. In this paper, three different qualitative measures are utilized.

Generational distance (GD) is a measure of the distance between the true $\left(P F_{\text {real }}\right)$ and generated Pareto front $\left(P F_{\text {algorithm }}\right)$. This metric of individual distance representing the distance is given by

$G D=\sqrt{\frac{1}{n_{p f}} \sum_{i=1}^{n_{p f}} d_{i}^{2}}$

Where $n_{p f}$ is the number of members in $P F_{\text {algorithm }}$ and $d_{i}$ is the Euclidean distance between the $i$ th member in $P F_{\text {algorithm }}$ and its nearest member in $P F_{\text {real }}$. A smaller value of GD implies better convergence.

The metric of spacing (S) gives an indication of how evenly the solutions are distributed along the discovered Pareto-front:

$S=\sqrt{\frac{1}{n_{p f}} \sum_{i=1}^{n_{p f}}\left(d_{i}-\bar{d}\right)^{2}} \quad, \quad \bar{d}=\frac{1}{n_{p f}} \sum_{i=1}^{n_{p f}} d_{i}$

Where $n_{p f}$ is the number of members in $P F_{\text {algorithm }}$ and $\mathrm{d}_{\mathrm{i}}$ is the Euclidean distance (in the objective space)

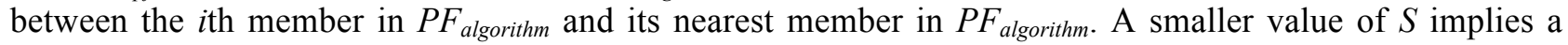
more uniform distribution of solutions in $P F_{\text {algorithm }}$.

The metric of maximum spread $(M S)$ measures how "well"' the $P F_{\text {real }}$ is covered by the $P F_{\text {algorithm }}$ through hyper-boxes formed by the extreme function values observed in the $P F_{\text {real }}$ and $P F_{\text {algorithm. }}$. It is defined as:

$M S=\left(\frac{1}{m} \sum_{i=1}^{m}\left(\frac{\min \left(f_{i}^{\max }, F_{i}^{\max }\right)-\max \left(f_{i}^{\min }, F_{i}^{\min }\right)}{F_{i}^{\max }-F_{i}^{\min }}\right)^{2}\right)^{0.5}$

Where $m$ is the number of objectives, $f_{i}^{\max }$ and $f_{i}^{\min }$ are the maximum and minimum of the $i$ th objective in $P F_{\text {algorithm }}$, respectively, and $F_{i}^{\max }$ and $F_{i}^{\min }$ are the maximum and minimum of the $i$ th objective in $P F_{\text {real }}$, respectively. A larger value of $M S$ implies a better spread of solutions.

\section{TEST PROBLEMS}

Four benchmark problems ZDT1, ZDT3, FON and POL are selected to examine the performance of the proposed algorithm. The test problems are detailed in table 1 [26-29].

Table.1: Benchmark test problems ZDT1, ZDT3, FON and POL

\begin{tabular}{lccc}
$\begin{array}{c}\text { Test } \\
\text { problem }\end{array}$ & Mathematical formulas & $\begin{array}{c}\text { Range of } \\
\text { variables }\end{array}$ & $\begin{array}{c}\text { Number of } \\
\text { variables }\end{array}$ \\
ZDT1 $\min \left\{\begin{array}{l}f_{l}(x)=x_{I} \\
f_{2}(x)=g(x)\left[1-\sqrt{\frac{x_{I}}{g(x)}}\right], g(x)=1+\frac{9}{n-1} \sum_{i=2}^{n} x_{i}\end{array}\right.$ & $0 \leq x \leq 1$ & 30 \\
\hline
\end{tabular}




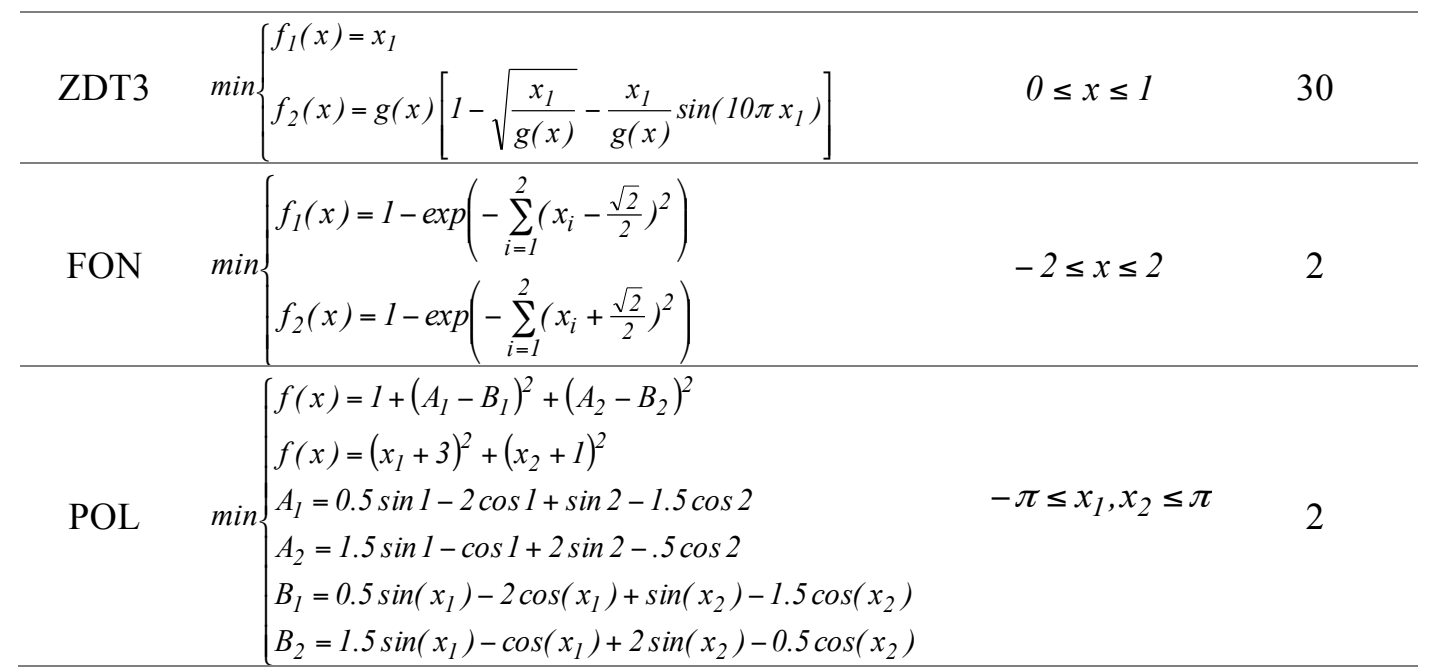

\section{CASE STUDY IN TCTP}

In this paper we will use multi-objective CSS algorithms mentioned in next sections to solve two wellknown time-cost optimization problems named as the Feng network activities and the Zheng network activities. The Feng network with 18 activities is one of the most well-known examples for time-cost optimization problem that has attracted the attention of many researchers [3]. Configuration and activities network options of Feng network are shown in Fig. 3 and Table 2 respectively. In addition to Feng activities network, another activities network with 7 activities introduced by Zheng considered as second example. Configuration and activities network options of Zheng network are shown in Fig. 4 and Table 3 respectively [10].

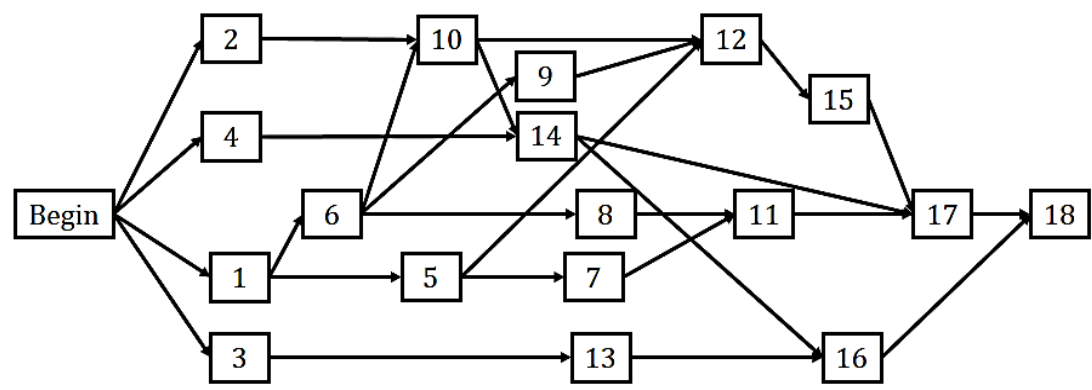

Fig. 3: Configuration of Feng network activities 
Table 2: Time, Cost, Number of execute options and Prerequisites of activities of Feng network

\begin{tabular}{|c|c|c|c|c|c|c|c|c|c|c|c|c|c|c|}
\hline No & $\operatorname{Pr}$ & Op & $\mathrm{Ti}$ & Cost & No & $\operatorname{Pr}$ & $\mathrm{Op}$ & $\mathrm{Ti}$ & Cost & No & $\operatorname{Pr}$ & Op & $\mathrm{Ti}$ & Cost \\
\hline \multirow{5}{*}{1} & & 1 & 14 & 2400 & \multirow{5}{*}{7} & \multirow{5}{*}{5} & & & & \multirow{5}{*}{13} & \multirow{5}{*}{3} & & & \\
\hline & & 2 & 15 & 2150 & & & 1 & 9 & 30000 & & & 1 & 14 & 4000 \\
\hline & - & 3 & 16 & 1900 & & & 2 & 15 & 24000 & & & 2 & 18 & 3200 \\
\hline & & 4 & 21 & 1500 & & & 3 & 18 & 22000 & & & 3 & 24 & 1800 \\
\hline & & 5 & 24 & 1200 & & & & & & & & & & \\
\hline \multirow{5}{*}{2} & \multirow{5}{*}{ - } & 1 & 15 & 3000 & \multirow{5}{*}{8} & \multirow{5}{*}{6} & & 14 & 220 & \multirow{5}{*}{14} & \multirow{5}{*}{$\begin{array}{c}4 \\
10\end{array}$} & & & \\
\hline & & 2 & 18 & 2400 & & & & 15 & 215 & & & 1 & 9 & 3000 \\
\hline & & 3 & 20 & 1800 & & & & 16 & 200 & & & 2 & 15 & 2400 \\
\hline & & 4 & 23 & 1500 & & & & 21 & 208 & & & 3 & 18 & 2200 \\
\hline & & 5 & 25 & 1000 & & & & 24 & 120 & & & & & \\
\hline \multirow{5}{*}{3} & \multirow{5}{*}{-} & & & & \multirow{5}{*}{9} & \multirow{5}{*}{6} & 1 & 15 & 300 & \multirow{5}{*}{15} & \multirow{5}{*}{12} & \multirow{5}{*}{$\begin{array}{l}1 \\
2\end{array}$} & \multirow{5}{*}{$\begin{array}{l}12 \\
16\end{array}$} & \multirow{5}{*}{$\begin{array}{l}4500 \\
3500\end{array}$} \\
\hline & & 1 & 15 & 4500 & & & 2 & 18 & 240 & & & & & \\
\hline & & 2 & 22 & 4000 & & & 3 & 20 & 180 & & & & & \\
\hline & & 3 & 33 & 3200 & & & 4 & 23 & 150 & & & & & \\
\hline & & & & & & & 5 & 25 & 100 & & & & & \\
\hline \multirow{3}{*}{4} & & 1 & 12 & 45000 & \multirow{3}{*}{10} & \multirow{3}{*}{2} & 1 & 15 & 450 & \multirow{3}{*}{17} & 11 & 1 & 14 & 4000 \\
\hline & - & 2 & 16 & 35000 & & & 2 & 22 & 400 & & 14 & 2 & 18 & 3200 \\
\hline & & 3 & 20 & 30000 & & & 3 & 33 & 320 & & 15 & 3 & 24 & 1800 \\
\hline \multirow{5}{*}{5} & \multirow{5}{*}{1} & 1 & 22 & 20000 & \multirow{5}{*}{11} & & & & & & & 1 & 20 & 3000 \\
\hline & & 1 & 24 & $\begin{array}{l}20000 \\
17500\end{array}$ & & & 1 & 12 & 450 & & & 2 & 22 & 2000 \\
\hline & & 2 & ס & 15000 & & 1 & 2 & 16 & 350 & 16 & 15 & 3 & 24 & 1750 \\
\hline & & 3 & $\begin{array}{l}20 \\
30\end{array}$ & 100000 & & & 3 & 20 & 300 & & & 4 & 28 & 1500 \\
\hline & & 4 & & 10000 & & & & & & & & 5 & 30 & 1000 \\
\hline & & & 14 & 40000 & & & 1 & 22 & 2000 & & & & 9 & \\
\hline 6 & 1 & 2 & 18 & 32000 & 12 & 9 & 2 & 24 & 1750 & 18 & 16 & 2 & 15 & 2400 \\
\hline & & 3 & 24 & 18000 & & 10 & $\begin{array}{l}3 \\
4\end{array}$ & $\begin{array}{l}28 \\
30\end{array}$ & $\begin{array}{l}1500 \\
1000\end{array}$ & & 17 & 3 & 18 & 2200 \\
\hline
\end{tabular}

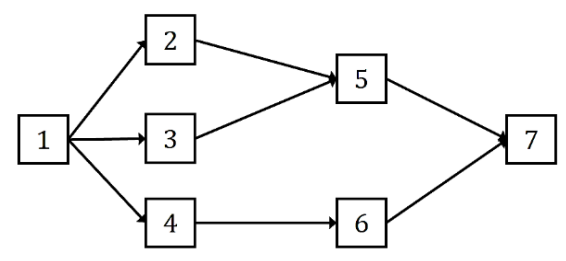

Fig. 4: Configuration of Zheng network activities

Table 3: Time, Cost, Number of execute options and Prerequisites of activities of Zheng network.

\begin{tabular}{ccccc|ccccc}
\hline No & Pr & Op & Ti & Cost & No & Pr & Op & Ti & Cost \\
\hline & & 1 & 14 & 23000 & & & 1 & 12 & 45000 \\
1 & - & 2 & 20 & 18000 & 4 & 1 & 2 & 16 & 35000 \\
& & 3 & 24 & 12000 & & & 3 & 20 & 30000 \\
\hline 2 & 1 & 1 & 15 & 3000 & 5 & 2 & 1 & 22 & 20000 \\
\hline
\end{tabular}




\section{RESULTS}

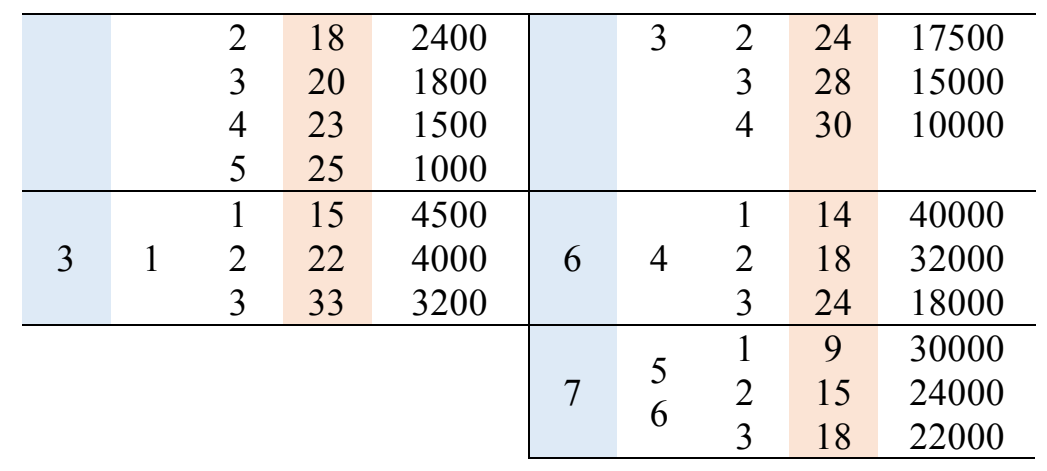

In order to illustrate the efficiency of our method, it is compared with two of most well-known multiobjective optimization algorithms named as NSGA_II and MOPSO. Parameters for comparison are include: mentioned qualitative measures (GD, S and MS) and the relative average running time of the algorithms (average running time of the algorithms divided on average running time of the SMOCSS algorithm). A random initial population is created for each of the 10 runs on each test problem. All of the algorithms are implemented in Matlab. The results obtained for test problems can be presented as follows:

\section{Test problem 1- ZDT1}

The comparison of results between the Pareto fronts produced by NSGA-II, MOPSO and SMOCSS of ZDT1 and the true Pareto front are shown in Fig. 5(a)-(c), respectively.
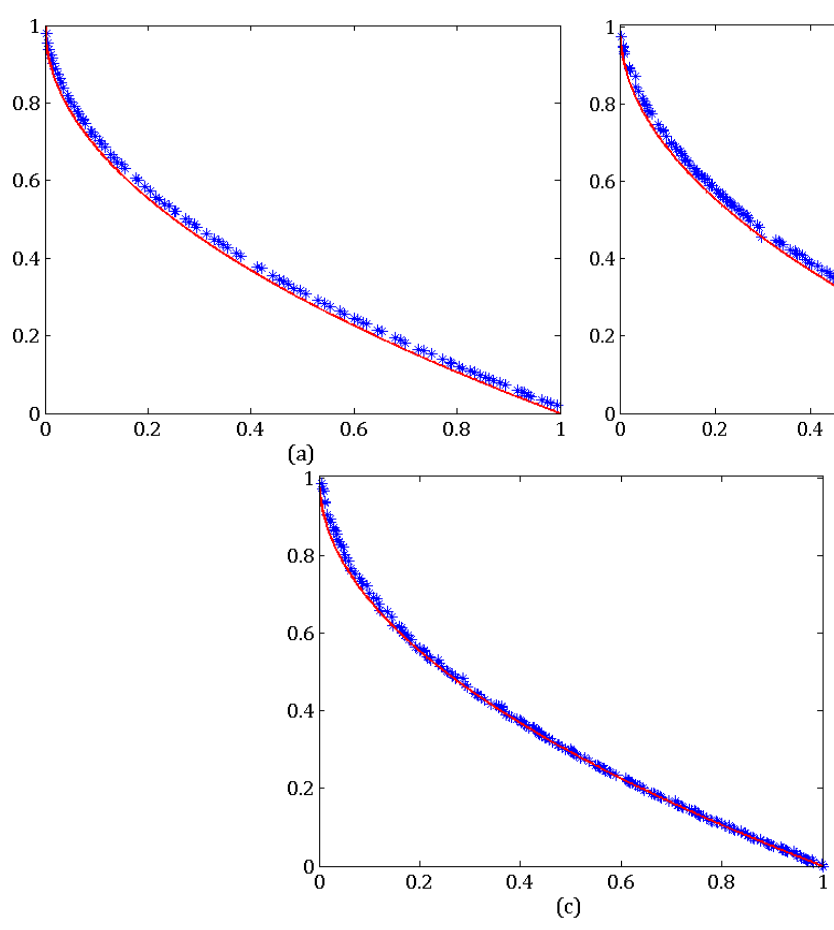

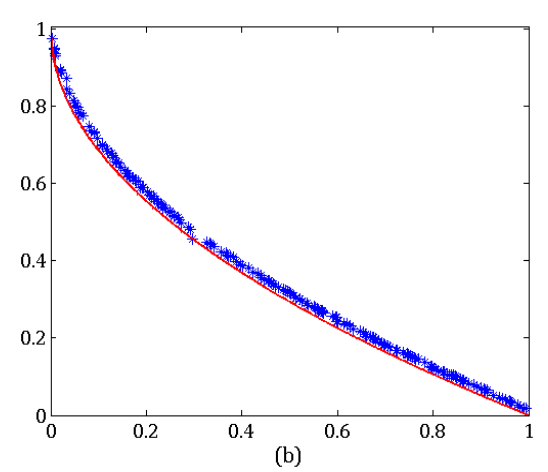

(b)

Fig.5: Pareto fronts produced by (a) NSGA-II, (b) MOPSO and (c) SMOCSS on test function ZDT1. 
From the Fig. 5, it can be observed that all algorithms are able to find solutions near the global Pareto front. The values of the three metrics for each algorithm are shown in Table 4. Considering all of the metrics from Table 4, it can be seen that new algorithm (SMOCSS) is the best among the three adopted algorithms.

Table.4: Results for ZDT1: The mean values of the three metrics and relative run time for each algorithm

\begin{tabular}{cccc}
\hline & NSGA_II & MOPSO & SMOCSS \\
\hline GD (mean) & 0.0149 & 0.1299 & 0.0058 \\
S (mean) & 0.0030 & 0.1261 & 0.0030 \\
MS (mean) & 0.9761 & 0.9879 & 1 \\
Relative Run Time & 5.41 & 1.35 & 1 \\
\hline
\end{tabular}

\section{Test problem 2- ZDT3}

The comparison of results between the Pareto fronts produced by NSGA-II, MOPSO and SMOCSS of ZDT3 and the true Pareto front are shown in Fig. 6(a)-(c), respectively.

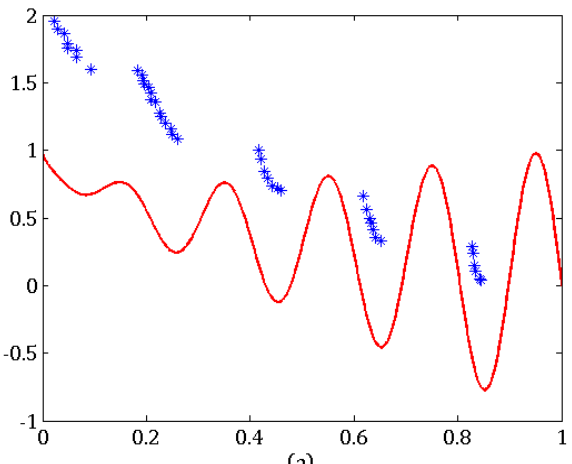

(a)

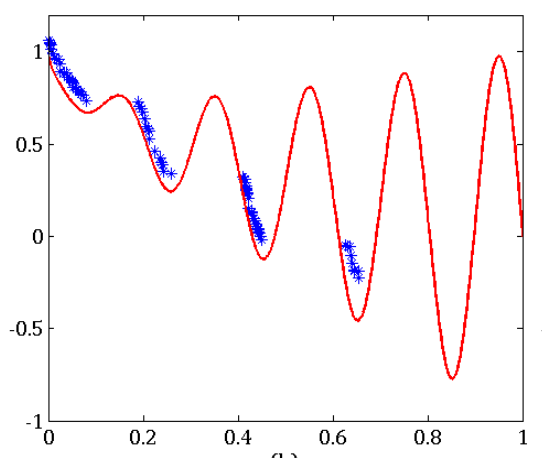

(b)

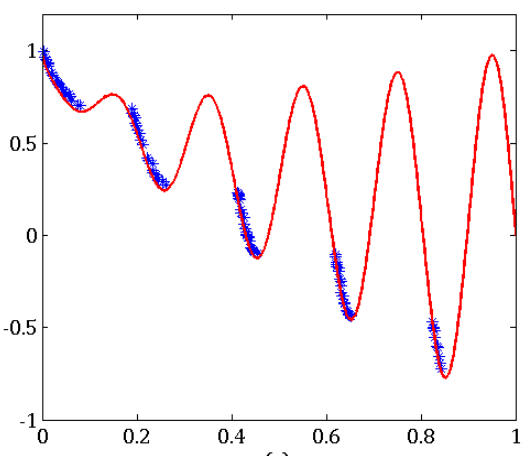

(c)

Fig.6: Pareto fronts produced by (a) NSGA-II, (b) MOPSO and (c) SMOCSS on test function ZDT3.

From the Fig.6 it can be revealed that the NSGA_II and the MOPSO algorithms are stuck in a local Pareto optimum, but the SMOCSS algorithm is able to evolve a diverse and well distributed near-optimal Pareto front by spending less run time. The values of the three metrics for each algorithm are shown in Table 5 . Considering all of the metrics from Table 53, it can be seen that new algorithm (SMOCSS) is the best among the three adopted algorithms.

Table.5: Results for ZDT3: The mean values of the three metrics and relative run time for each algorithm

\begin{tabular}{cccc}
\hline & NSGA_II & MOPSO & SMOCSS \\
\hline GD (mean) & 0.4389 & 0.211 & 0.0079 \\
S (mean) & 0.2917 & 0.0084 & 0.0039 \\
MS (mean) & 0.6978 & 0.6713 & 0.9102 \\
Relative Run Time & 4.89 & 1.13 & 1 \\
\hline
\end{tabular}

Test problem 3- FON

The comparison of results between the Pareto fronts produced by NSGA-II, MOPSO and SMOCSS of FON and the true Pareto front are shown in Fig. 7(a)-(c), respectively.

Table.6: Results for FON: The mean values of the three metrics and relative run time for each algorithm 


\begin{tabular}{cccc}
\hline & NSGA_II & MOPSO & SMOCSS \\
\hline GD (mean) & 0.0016 & 0.0006 & 0.008 \\
S (mean) & 0.0012 & 0.0004 & 0.0005 \\
MS (mean) & 0.9960 & 0.9994 & 0.9991 \\
Relative Run Time & 4.53 & 1.09 & 1 \\
\hline
\end{tabular}

From the Fig.7 it can be seen that all algorithms are able to evolve a diverse and well distributed nearoptimal Pareto front for this problem but the computation time required for NSGA_II and MOPSO algorithms are more than the run time required for SMOCSS algorithm. So that even the NSGA_II run time about 4.5 times more that the same for our algorithm. The values of the three metrics for each algorithm are shown in Table 6. Considering all of the metrics from Table 6, it can be seen that all algorithms are usable to optimizing this problem.
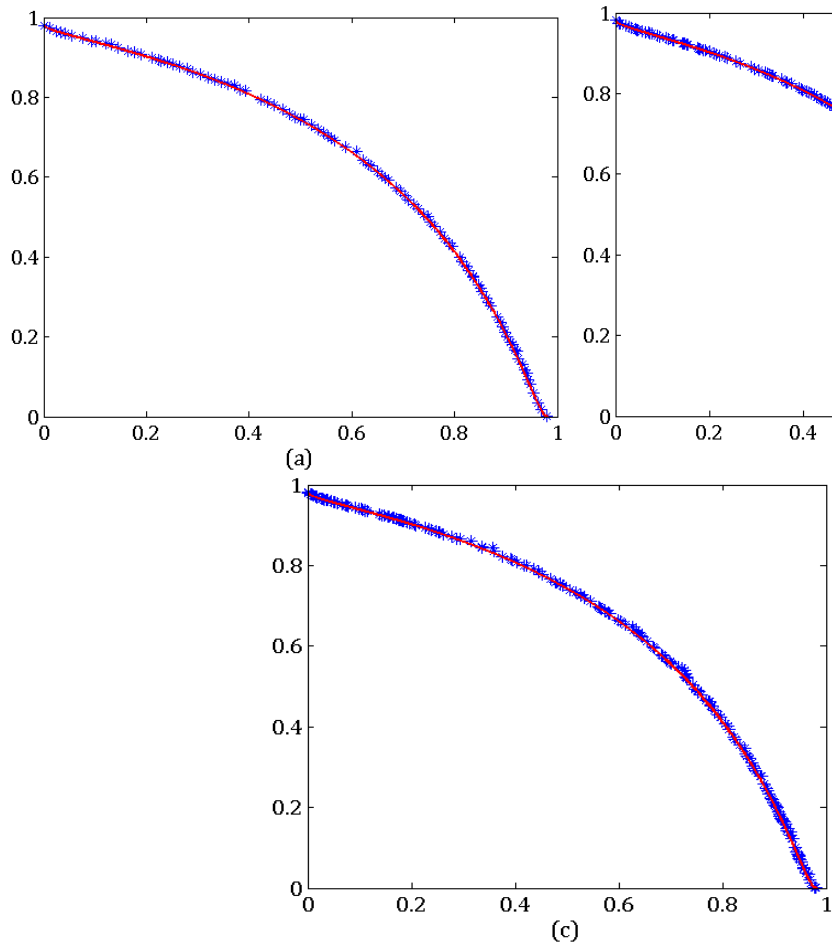

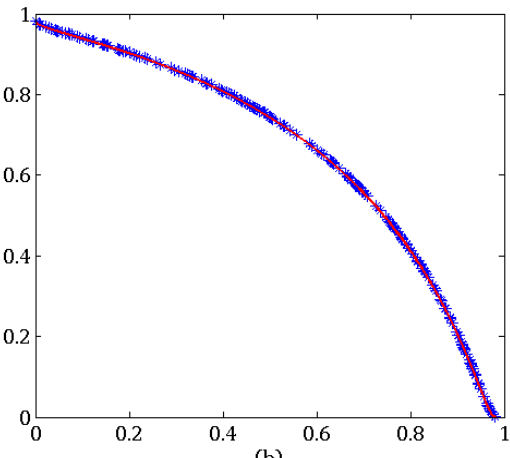

(b)

Fig.7: Pareto fronts produced by (a) NSGA-II, (b) MOPSO and (c) SMOCSS on test function FON.

\section{Test problem 4- POL}

The comparison of results between the Pareto fronts produced by NSGA-II, MOPSO and SMOCSS of POL and the true Pareto front are shown in Fig. 8(a)-(c), respectively. From the Fig. 8 it can be seen that similar to the previous problem, all algorithms are able to evolve an almost diverse and well distributed near-optimal Pareto front for this problem but the computation time required for NSGA_II and MOPSO algorithms are more than the run time required for SMOCSS algorithm. So that even the NSGA II run time about 4 times more that the same for our algorithm. The values of the three metrics for each algorithm are shown in Table 
7. Considering all of the metrics from Table 7, it can be seen that NSGA_II is better than MOPSO but it requires more run time and both of this two algorithms are not as good as SMOCSS.

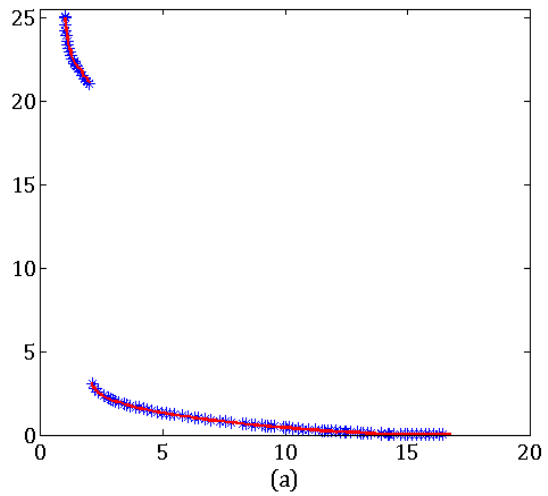

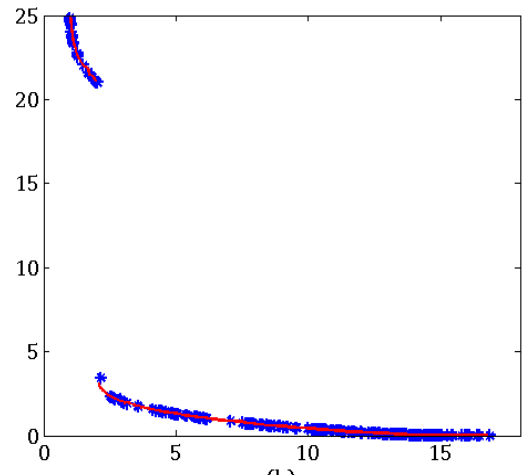

(b)

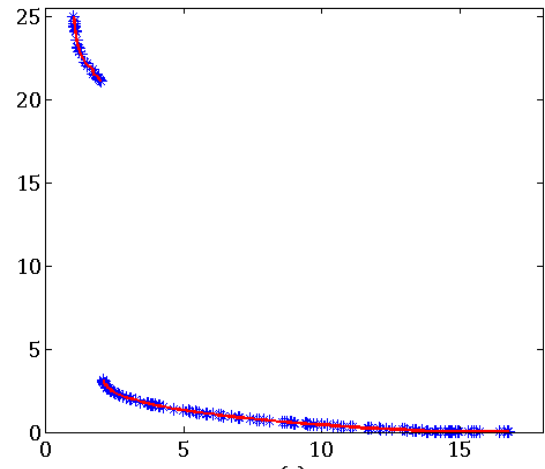

(c)

Fig.8: Pareto fronts produced by (a) NSGA-II, (b) MOPSO and (c) SMOCSS on test function POL.

Table.7: Results for POL: The mean values of the three metrics and relative run time for each algorithm

\begin{tabular}{cccc}
\hline & NSGA_II & MOPSO & SMOCSS \\
\hline GD (mean) & 0.0493 & 0.0701 & 0.0277 \\
S (mean) & 0.0210 & 0.0478 & 0.0121 \\
MS (mean) & 0.9900 & 0.9908 & 0.9961 \\
Relative Run Time & 4.16 & 1.01 & 1 \\
\hline
\end{tabular}

The Feng and the Zheng activities network, mentioned in section 3, were solved by NSGA-II, MOPSO, MAWA-CSS and SMOCSS algorithms and the results were compared in the number of non-dominated solutions and relative run time of algorithms. All of the algorithms are implemented in Matlab and each problem was solved 10 times and mean of results was recorded. Table 8 shows the number of non-dominated solutions and relative run time of algorithms.

Table 8: The number of non-dominated solutions and relative run time of algorithms.

\begin{tabular}{|c|c|c|c|c|c|c|c|c|}
\hline \multirow[b]{2}{*}{ Network } & \multicolumn{4}{|c|}{$\begin{array}{l}\text { the number of non- } \\
\text { dominated solutions }\end{array}$} & \multicolumn{4}{|c|}{ Run Time } \\
\hline & $\begin{array}{l}Z \\
Z \\
\Omega \\
\Omega \\
\stackrel{D}{D} \\
=\end{array}$ & $\begin{array}{l}3 \\
0 \\
0 \\
0 \\
0\end{array}$ & 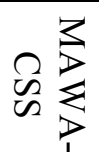 & 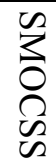 & $\begin{array}{l}Z \\
\mathscr{D} \\
\Omega \\
\stackrel{D}{1} \\
\vdots \\
=\end{array}$ & $\begin{array}{l}3 \\
0 \\
0 \\
0 \\
0\end{array}$ & $\underset{\infty}{\infty} \sum_{1}^{3}$ & $\begin{array}{l}\infty \\
3 \\
\Omega \\
\Omega \\
\Omega\end{array}$ \\
\hline Feng & 44 & 44 & 44 & 44 & 2.2 & 2.1 & 1.1 & 1 \\
\hline Zheng & 23 & 23 & 23 & 23 & 2.8 & 1.9 & 1.2 & 1 \\
\hline
\end{tabular}

It can be seen from table 8 that SMOCSS algorithm is faster than other algorithms and also is able to find all solutions in pareto set and MAWA-CSS is not as good as SMOCSS algorithm but is faster than MOPSO and NSGA-II. Furthermore, sometimes the MOPSO and the NSGA-II could not find all solutions of Feng 
activities network.

As a result of solving the Zheng and Feng activities networks with SMOCSS Following solutions achieved (table 9 and table 10).

Table 9: Results of solving the Zheng activities networks with SMOCSS

\begin{tabular}{cccccccc}
\hline Time & Cost & Time & Cost & Time & Cost & Time & Cost \\
\hline 60 & 143500 & 67 & 123500 & 78 & 107500 & 92 & 98300 \\
61 & 142500 & 68 & 118500 & 81 & 106900 & 94 & 97800 \\
62 & 140000 & 71 & 117900 & 83 & 105500 & 95 & 97500 \\
63 & 131000 & 73 & 117300 & 84 & 101500 & 97 & 97000 \\
65 & 130400 & 74 & 112500 & 87 & 99500 & 105 & 96200 \\
66 & 128500 & 77 & 110500 & 90 & 98900 & & \\
\hline
\end{tabular}

Table 10: Results of solving the Feng activities networks with SMOCSS

\begin{tabular}{cccccccc}
\hline Time & Cost & Time & Cost & Time & Cost & Time & Cost \\
\hline 100 & 133320 & 111 & 106020 & 125 & 102820 & 142 & 100870 \\
101 & 128320 & 112 & 105770 & 126 & 102570 & 143 & 100770 \\
102 & 128070 & 114 & 105270 & 128 & 102320 & 145 & 100570 \\
103 & 127820 & 115 & 105020 & 131 & 102170 & 148 & 100270 \\
104 & 120320 & 116 & 104770 & 132 & 101970 & 151 & 100070 \\
105 & 120070 & 118 & 104470 & 133 & 101820 & 154 & 100010 \\
106 & 119820 & 119 & 104220 & 134 & 101570 & 156 & 99950 \\
107 & 119770 & 120 & 103970 & 137 & 101510 & 158 & 99900 \\
108 & 119270 & 121 & 103820 & 138 & 101470 & 159 & 99870 \\
109 & 119020 & 122 & 103570 & 139 & 101170 & 161 & 99820 \\
110 & 106270 & 124 & 103070 & 140 & 100970 & 169 & 99740 \\
\hline
\end{tabular}

Fig. 9 and Fig. 10 shows all results of solving the Zheng and Feng activities networks by SMOCSS respectively.

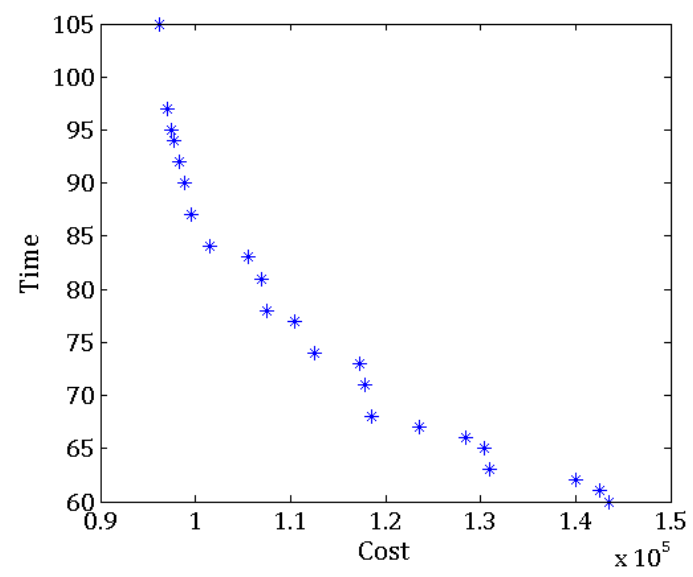

Fig.9: All results of solving the Zheng activities networks by SMOCSS. 


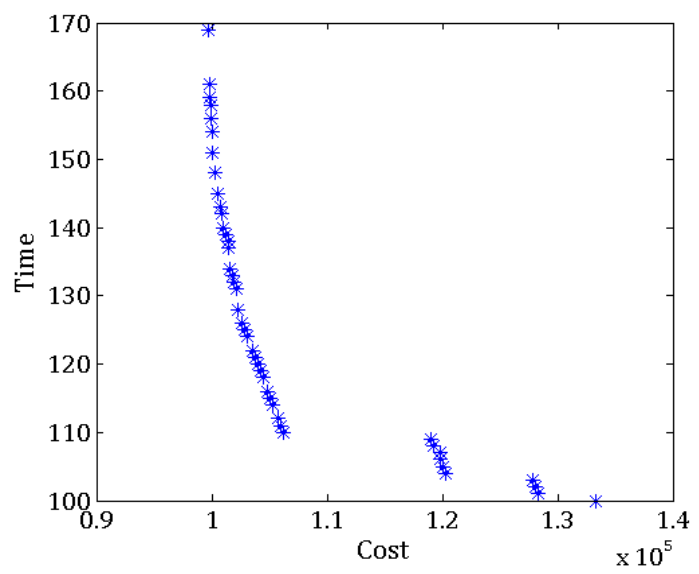

Fig.10: All results of solving the Feng activities networks by SMOCSS.

\section{CONCLUSION}

In this paper, a new multi-objective optimization algorithm, named as SMOCSS, is proposed. The proposed algorithm is based on the recently developed algorithm, charge system search (CSS). In this approach, each particle creates an electrical field around itself and consequently affects other particles by a force which is a result of this attraction field. By this pattern, better particles attract worse particles. The overall structure of this new algorithm is similar to the MOPSO and to determine the Charge magnitude of particles a new simple method is introduced. A comparative study of SMOCSS and two state-of-the-art MO algorithms on four benchmark test problems is presented. The results of applying three performance metrics clearly indicate that SMOCSS is competitive and even outperforms most of the selected MO algorithms. The comparison of the computational time shows that SMOCSS requires less computer time than the selected MO algorithms on some of the selected benchmark test problems.

Construction time-cost trade-off problems are large scale two-objective optimization problems and finding out all solutions in Pareto set with spending reasonable time is very important. By generalizing the wellknown Charged Search System (CSS), with using of a new method (SMOCSS), to optimize multi-objective problems, not only the optimal solution could be found in reasonable time, but also the Pareto front could be defined that will provide more effective information and basis for further and correct decision-making. The result of proposed model is compared with MAWA-CSS algorithm, as another multi-objective optimizer, and also NSGA-II and it is concluded that current procedure of modeling TCTP has several advantages especially in terms of generating better solutions and consuming less time for solving the problem.

\section{REFRENCES}

T.J Hindelang, J.F Muth, A dynamic programming algorithm for decision CPM networks. Operat Res, pp. 225-241, 1979.

Elmaghraby, S. E. 'Resource allocation via dynamic programming in activity networks', Eur. J. Operational Res., Vol. 64, pp. 199-215. 1993.

Feng, C.W., Liu, L. and Burns, S.A.'Using genetic algorithms to solve construction time-cost trade-off problems', Journal of Computing in Civil Engineering, ASCE, Vol. 11, No. 3, pp. 184-189. 1997.

Kelly, James, E. Jr. 'Critical path planning and scheduling: mathematical basis', Oper. Res., Vol. 9 no. 3, pp. 167-179. 1961. 
Hendrickson, C. and Au,T. Project Management for Construction, Prentice-Hall, Inc, Englewood Cliffs, N.J. 1989.

Butcher, W.S. 'Dynamic programming for project cost-time curve', Journal of Construction Division, ASCE, Vol. 93 (C01), pp. 59-73. 1967.

Robinson, D. R. 'A dynamic programming solution to cost-time tradeoff for CPM', Mgmt. Sci., Vol. 22, No. 2, pp. 158-166. 1975

Liu, L., Burns,S.A. and Feng, C. 'Construction time-cost trade-off analysis using LP/IP', Journal of Construction Engineering and Management, ASCE, Vol. 121 (4), pp. 446-454. 1995.

Burns, S.A., Liu, L. and Feng, C.W. 'The LP/IP hybrid method for construction time-cost trade off analysis', Constr. Manag. Econ., Vol. 14, No. 3, pp. 265-276. 1996.

Zheng, D. X.M., Ng, S.T. and Kumaraswamy, M.M. 'Applying a genetic algorithm-based multi-objective approach for time-cost optimization', Journal of Construction Engineering and Management, Vol. 130, No. 2, pp. 168-176. 2004.

Carlos A. Coello Coello, David A. Van Veldhuizen, Gary B. Lamont. Evolutionary Algorithms for Solving Multi-Objective Problems. Kluwer Academic Publishers, Boston. ISBN 0-3064-6762-3. 2002.

David A. Van Veldhuizen, Gary B. Lamont. Multi-Objective Evolutionary Algorithms: Analyzing the Stateof-the-Art. Evolutionary Computation, 7(3):1-26, 2000.

Yang, I.T. 'Using elitist particle swarm optimization to facilitate bicriterion time-cost trade-off analysis', J. Constr. Eng. Manage., Vol. 133, No. 7, pp. 498-505. 2007.

Geem, Z. W. Multiobjective optimization of time-cost trade-off using harmony search, Journal of Construction Engineering and Management, ASCE, Vol. 136, No. 6, pp. 711- 716. 2010.

$\mathrm{Ng}$, S. T. and Zhang, Y. Optimizing construction time and cost using ant colony optimization approach, Journal of Construction Engineering and Management, ASCE, Vol. 134, No. 9, pp. 721-728. 2008.

Daisy X. M. Zheng, S. Thomas Ng, and Mohan M. Kumaraswamy. "Applying Pareto Ranking and Niche Formation to Genetic Algorithm-Based Multiobjective Time-Cost Optimization.” J. Construction. Eng. Manage. 131(1), 81-91. 2005.

Kaveh A, Talatahari S. A novel heuristic optimization method: charged system search. Acta Mech; 213 : 267-89. 2010.

Kaveh, A., \& Talatahari, S. Optimal design of skeletal structures via the charged system search algorithm. Structural and Multidisciplinary Optimization (37), 893-911. 2010.

Kaveh, A., \& Talatahari, S. Charged system search for optimum grillage systems design using the LRFDAISC code. Journal of Constructional Steel Research (66), 767-771. 2010.

Coello Coello, C. A., Van Veldhuizen, D. A., \& Lamont, G. B. Evolutionary algorithms for solving multi objective problems. New York: Kluwer Academic Publishers. 2002.

Qi, K., Lei, W., \& Qidi, W. A novel self-organizing particle swarm optimization based on gravitation field model. In Proceeding of the American Control Conference, New York (pp. 528-533). 2007.

Coello Coello, C. A., Pulido, G. T., \& Lechuga, M. S. handling multiple objectives with particle swarm optimization. IEEE Transaction on Evolutionary Computation, 8(3), 256-279. 2004.

Zitzler, E., Laumanns, M., \& Thiele, L. SPEA2: Improving the strength Pareto evolutionary algorithm. Zurich, Switzerland: Swiss Federal Institute Technology. 2001.

Zitzler, E., Deb, K., \& Thiele, L. Comparison of multi-objective evolutionary algorithms: Empirical results. Evolutionary Computation, 8(2), 173-195. 2000

Deb, K., Pratap, A., Agarwal, S., \& Meyarivan, T. A fast and elitist multi-objective genetic algorithm: NSGA-II. IEEE Transaction on Evolutionary Computation, 6(2), 182-197. 2002.

Zitzler, E., \& Thiele, L. Multi objective evolutionary algorithms: A comparative case study and the strength Pareto approach. IEEE Transactions on Evolutionary Computation, 3(4), 257-271. 1999

Tsai, S. J., Sun, T. Y., Liu, C. C., Hsieh, S. T., Wu, W. C., \& Chiu, S. Y. An improved multi-objective particle swarm optimizer for multi-objective problems. Expert Systems with Applications, 37(8), 58725886. 2010. 
Chen, J., Lin, Q., \& Ji, Z. A hybrid immune multi objective optimization algorithm. European Journal of Operational Research (204), 294-302. 2010.

Deb, K. Multi objective optimization using evolutionary algorithms. Wiley, U.K: Chichester. 2001. 